\title{
Lattice Gauge Theories and String Dynamics in Rydberg Atom Quantum Simulators
}

\author{
Federica M. Surace, ${ }^{1,2}$ Paolo P. Mazza, ${ }^{1,3}$ Giuliano Giudici, ${ }^{1,2,3}$ Alessio Lerose, ${ }^{1,3}$ \\ Andrea Gambassi, ${ }^{1,3}$ and Marcello Dalmonte ${ }^{1,2}$ \\ ${ }^{1}$ SISSA-International School for Advanced Studies, via Bonomea 265, 34136 Trieste, Italy \\ ${ }^{2}$ ICTP-International Center for Theoretical Physics, Strada Costiera 11, 34151 Trieste, Italy \\ ${ }^{3}$ INFN, Sezione di Trieste, via Bonomea 265, 34136 Trieste, Italy
}

(Received 5 April 2019; revised manuscript received 1 February 2020; accepted 10 March 2020; published 21 May 2020)

\begin{abstract}
Gauge theories are the cornerstone of our understanding of fundamental interactions among elementary particles. Their properties are often probed in dynamical experiments, such as those performed at ion colliders and high-intensity laser facilities. Describing the evolution of these strongly coupled systems is a formidable challenge for classical computers and represents one of the key open quests for quantum simulation approaches to particle physics phenomena. In this work, we show how recent experiments done on Rydberg atom chains naturally realize the real-time dynamics of a lattice gauge theory at system sizes at the boundary of classical computational methods. We prove that the constrained Hamiltonian dynamics induced by strong Rydberg interactions maps exactly onto the one of a U(1) lattice gauge theory. Building on this correspondence, we show that the recently observed anomalously slow dynamics corresponds to a string-inversion mechanism, reminiscent of the string breaking typically observed in gauge theories. This underlies the generality of this slow dynamics, which we illustrate in the context of one-dimensional quantum electrodynamics on the lattice. Within the same platform, we propose a set of experiments that generically show long-lived oscillations, including the evolution of particleantiparticle pairs, and discuss how a tunable topological angle can be realized, further affecting the dynamics following a quench. Our work shows that the state of the art for quantum simulation of lattice gauge theories is at 51 qubits and connects the recently observed slow dynamics in atomic systems to archetypal phenomena in particle physics.
\end{abstract}

DOI: 10.1103/PhysRevX.10.021041

\section{INTRODUCTION}

Lattice gauge theories (LGTs) [1] represent one of the most successful frameworks for describing fundamental interactions within the standard model of particle physics. Numerical simulations of their Euclidean formulation [2] have shed light on paradigmatic equilibrium properties of strong interactions, including the low-lying spectrum of quantum chromodynamics [3], and the nature of its phase diagram $[4,5]$. Nonequilibrium properties, instead, are a notable challenge [6], due to the lack of generically applicable methods to simulate the real-time dynamics of extended, strongly interacting systems [7]. This has stimulated an intense theoretical activity aimed at quantum simulating LGTs via atomic quantum systems [8-10], leading to

Published by the American Physical Society under the terms of the Creative Commons Attribution 4.0 International license. Further distribution of this work must maintain attribution to the author(s) and the published article's title, journal citation, and DOI.
Subject Areas: Atomic and Molecular Physics,

Condensed Matter Physics,

Particles and Fields,

Quantum Information

the first door-opener experimental realization in a system of four trapped ions [11]. While such quantum simulators have already challenged the most advanced computational techniques for studying condensed-matter motivated models $[12,13]$, there is presently no experimental evidence that atomic systems can be used to simulate LGTs at large scales, nor that they can display physical phenomena with a direct counterpart in LGTs. This limitation stems from the very characteristic aspect that distinguishes LGTs from other statistical mechanics models, i.e., the presence of local constraints on the possible configurations, in the form of a Gauss law, which cannot be easily implemented in actual experimental realizations $[8,9]$.

Here, we show that $(1+1)$-dimensional LGTs akin to quantum electrodynamics are naturally realized in state-ofthe-art experiments with Rydberg atom arrays $[14,15]$. In particular, we show how the dynamics of Rydberg excitations in these chains is exactly mapped onto a spin- $1 / 2$ quantum link model (QLM), a U(1) LGT where the gauge fields span a finite-dimensional Hilbert space, equivalent to a lattice Schwinger model in the presence of a topological 
(a) Rydberg atom chain

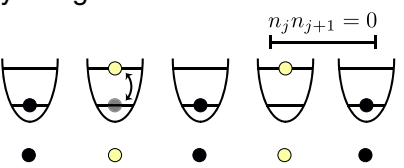

(d)

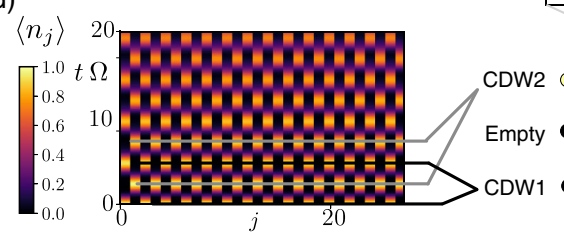

(c) Mapping

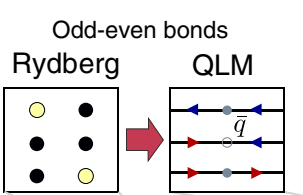

Even-odd bonds

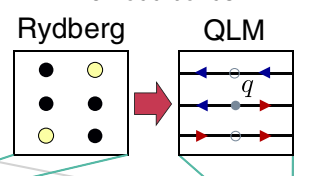

(b) Quantum link model

\section{(e)}
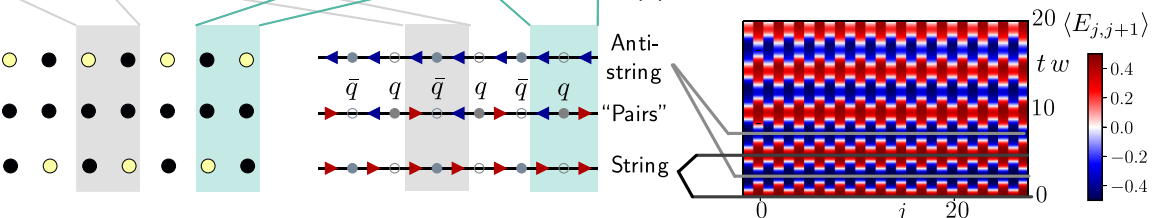

FIG. 1. Gauge-theory interpretation of Rydberg atom quantum simulations. (a) Schematics of a Rydberg atom chain. Each potential well of the optical lattice hosts a single atom, which can be in either the ground (black) or excited Rydberg (yellow) state. These two levels are coupled by a laser field. The Rydberg blockade prevents the simultaneous excitations of neighboring atoms. (b) Degrees of freedom of a U(1) LGT in the spin-1/2 quantum link model (QLM) formulation. Gauge fields are represented by spin variables residing on links. Matter fields are represented by Kogut-Susskind fermions: an occupied site corresponds to the vacuum on odd sites and to a quark $q$ on even sites. An empty site, instead, corresponds to the vacuum on even sites and to an antiquark $\bar{q}$ on odd sites. (c): Mapping between Rydberg-blockaded states and configurations of the electric field constrained by the Gauss law in the QLM. Because of the staggered electric charge, the allowed configurations of the electric field depend on the link, as illustrated. The two so-called chargedensity wave configurations "CDW1" and "CDW2" of the Rydberg atom arrays are mapped onto the "string" and "antistring" states, respectively, characterized by uniform rightward or leftward electric fluxes. The empty configuration with all Rydberg atoms in their ground state is mapped to a state filled by adjacent particle-antiparticle pairs. (d) Time evolution of the Rydberg array governed by the effective Hamiltonian $H_{\mathrm{FSS}}$ in Eq. (2), starting from the CDW1 state. The plot shows the space and time resolved population $\left\langle n_{j}\right\rangle$ of the excited Rydberg atoms. (e) Evolution of the expectation value of the electric field operator $\hat{E}_{j, j+1}$ in the QLM. These dynamics map exactly onto the ones shown in (d) via the mapping illustrated in (c). The thin lines highlight the oscillation between CDW1, CDW2 [left, bottom of (c)] or string and antistring (right) states. In these simulations, $L=24$ and $\delta=m=0$.

term [16]. The key element of our mapping, which is schematically illustrated in Fig. 1, is that gauge invariance has a natural counterpart in the Rydberg blockade mechanism, which constrains the Hilbert space in the same way as Gauss law does in gauge theories. This provides an immediate interpretation of the recent experiment with Rydberg-blockaded atom arrays in Ref. [14] as the first large-scale quantum simulation of a LGT at the edge of classical computational methods [7].

From a theoretical viewpoint, the mapping offers a hitherto unexplored perspective on the anomalously slow relaxation recently observed in experiments: the long-lived oscillations in the population of excited Rydberg atoms correspond to a string inversion, a phenomenon which is directly tied to string breaking $[6,17,18]$ prototypical of gauge theories including dynamical matter [cf. Figs. 1(d) and 1(e)]. The mapping indicates that this phenomenon has a natural interpretation in the LGT framework and suggests the occurrence of slow dynamics in other $\mathrm{U}(1)$ gauge theories, such as higher-spin QLMs [19], Higgs theories [20,21], and the Schwinger model [22,23]. These theories have been widely discussed in the context of Schwinger pair production taking place at high-intensity laser facilities, thus providing a highly unexpected, direct link between apparently unrelated experimental platforms [18,24-27].

We discuss the generality of this type of quantum evolution by extending our analysis to other relevant instances of "slow dynamics," characterized by the absence of relaxation on all timescales corresponding to any microscopic coupling present in the system. As initial states, we focus on those consisting of particle-antiparticle pairs, corresponding to regular configurations of the Rydberg atom arrays with localized defects, which are accessible within the setup of Ref. [14]. We show that these defects propagate ballistically with long-lived coherent interference patterns. This behavior is found to be governed by special bands of highly excited eigenstates characterized by a regularity in the energy-momentum dispersion relation. These findings open up a novel perspective which complements and extends toward gauge theories recent approaches to slow relaxation in Rydberg-blockaded atomic chains [28-33].

\section{RYDBERG ATOM ARRAYS}

We are interested here in the dynamics of a one-dimensional array of $L$ optical traps, each of them hosting a single atom, as schematically illustrated in Fig. 1(a). The atoms are trapped in their electronic ground state (black circle), denoted by $|\downarrow\rangle_{j}$, where $j$ numbers the trap. These ground states are quasiresonantly coupled to a single Rydberg state, i.e., a highly excited electronic level, denoted by $|\uparrow\rangle_{j}$. The dynamics of this chain of qbits $\left\{|\uparrow, \downarrow\rangle_{j}\right\}_{j=1, \ldots, L}$ is governed by the following Ising-type Hamiltonian [13,34]: 


$$
\hat{H}_{\mathrm{Ryd}}=\sum_{j=1}^{L}\left(\Omega \hat{\sigma}_{j}^{x}+\delta \hat{\sigma}_{j}^{z}\right)+\sum_{j<\ell=1}^{L} V_{j, \ell} \hat{n}_{j} \hat{n}_{\ell},
$$

where $\hat{\sigma}_{j}^{\alpha}$ are Pauli matrices at site $j$, the operator $\hat{n}_{j}=$ $\left(\hat{\sigma}_{j}^{z}+1\right) / 2$ signals the presence of a Rydberg excitation at site $j, 2 \Omega$ and $2 \delta$ are the Rabi frequency and the detuning of the laser excitation scheme, respectively, and $V_{j, \ell}$ describes the interactions between atoms in their Rydberg states at sites $(j, \ell)$. For the cases of interest here, this interaction is strong at short distances and decays as $1 /|j-\ell|^{6}$ at large distances. The dynamics described by $\hat{H}_{\text {Ryd }}$ has already been realized in several experiments utilizing either optical lattices or optical tweezers [14,15,35]. In particular, Ref. [14] investigated the case in which $V_{j, j+1}$ is much larger than all other energy scales of the system, resulting in the so-called Rybderg blockade effect: atoms on neighboring sites cannot be simultaneously excited to the Rydberg state, hence the constraint $\hat{n}_{j} \hat{n}_{j+1}=0$.

In this regime, the resulting Hamiltonian-introduced by Fendley, Sengupta, and Sachdev (FSS) in Ref. [36]—is

$$
\hat{H}_{\mathrm{FSS}}=\sum_{j=1}^{L}\left(\Omega \hat{\sigma}_{j}^{x}+2 \delta \hat{n}_{j}\right),
$$

where we neglect longer-range terms which do not affect qualitatively the dynamics. $\hat{H}_{\text {FSS }}$ acts on the constrained Hilbert space without double occupancies on nearestneighbor sites, as illustrated in Fig. 1(a). As we show below, the direct connection between Rydberg atomic systems and gauge theories is indeed provided by this constraint at the level of the Hilbert space.

\section{RYDBERG BLOCKADE AS A GAUGE SYMMETRY CONSTRAINT}

We establish here the exact mapping between the FSS Hamiltonian in Eq. (2) governing the dynamics of the Rydberg atom quantum simulator in Ref. [14] and a U(1) LGT. The latter describes the interaction between fermionic particles, denoted by $\hat{\Phi}_{j}$ and residing on the lattice site $j$, mediated by a $\mathrm{U}(1)$ gauge field, i.e., the electric field $\hat{E}_{j, j+1}$, defined on lattice bonds, as depicted in Fig. 1(b). We use here Kogut-Susskind (staggered) fermions [23], with the conventions that holes on odd sites represent antiquarks $\bar{q}$, while particles on even sites represent quarks $q$. Their dynamics is described by

$$
\begin{aligned}
\hat{H}= & -w \sum_{j=1}^{L-1}\left(\hat{\Phi}_{j}^{\dagger} \hat{U}_{j, j+1} \hat{\Phi}_{j+1}+\text { H.c. }\right) \\
& +m \sum_{j=1}^{L}(-1)^{j} \hat{\Phi}_{j}^{\dagger} \hat{\Phi}_{j}+J \sum_{j=1}^{L-1} \hat{E}_{j, j+1}^{2},
\end{aligned}
$$

where the first term provides the minimal coupling between gauge and matter fields through the parallel transporter $\hat{U}_{j, j+1}$ with $\left[\hat{E}_{j, j+1}, \hat{U}_{j, j+1}\right]=\hat{U}_{j, j+1}$, the second term is the fermion mass, and the last one is the electric field energy. The generators of the U(1) gauge symmetry are defined as

$$
\hat{G}_{j}=\hat{E}_{j, j+1}-\hat{E}_{j-1, j}-\hat{\Phi}_{j}^{\dagger} \hat{\Phi}_{j}+\frac{1-(-1)^{j}}{2},
$$

and satisfy $\left[\hat{H}, \hat{G}_{j}\right]=0$, so that gauge invariant states $|\Psi\rangle$ satisfy Gauss law $\hat{G}_{j}|\Psi\rangle=0$ for all values of $j$. Restricting the dynamics to their subspace is by far the most challenging task for quantum simulators.

Different formulations of U(1) LGTs are obtained for different representations of gauge degrees of freedom $\hat{E}_{j, j+1}$. While in the standard Wilsonian formulation-i.e., the lattice Schwinger model-they span infinitedimensional Hilbert spaces, here we first focus on the U(1) QLM formulation [19,37], where they are represented by spin variables, i.e., $\hat{E}_{j, j+1}=\hat{S}_{j, j+1}^{z}$ and $\hat{U}_{j, j+1}=\hat{S}_{j, j+1}^{+}$, so that $\left[\hat{E}_{j, j+1}, \hat{S}_{j, j+1}^{+}\right]=\hat{S}_{j, j+1}^{+}$. As noted in Ref. [38], this formulation is particularly suited for quantum simulation purposes.

In the following, we consider the QLM with spin $S=1 / 2$, in which all the possible configurations of the electric field have the same electrostatic energy, rendering the value of $J$ inconsequential; in Sec. IV B we show that this model is equivalent to the lattice Schwinger model in the presence of a $\theta$ angle with $\theta=\pi$ [39]. The Hilbert space structure following Gauss law is particularly simple in this case [38]: as depicted in Fig. 1(c), for each block along the chain consisting of two electric fields neighboring a matter field at site $j$, there are only three possible states, depending on the parity of $j$. In fact, in a general $(1+1)$-dimensional U(1) LGT, the configuration of the electric field along the chain determines the configuration of the charges via the Gauss law. Accordingly, $\hat{H}$ in Eq. (3) can be recast into a form in which the matter fields $\hat{\Phi}_{j}$ are integrated out.

We now provide a transformation which maps exactly the latter form into the FSS Hamiltonian Eq. (2). The correspondence between the two Hilbert spaces is realized by identifying, alternately on odd and even lattice sites, the computational basis configurations of the atomic qubits allowed by the Rydberg blockade with the classical configurations of the electric field allowed by the Gauss law [see Fig. 1(c)]. In terms of the two Hamiltonians, Eqs. (2) and (3), this unitary transformation consists in identifying the operators $\hat{\sigma}_{j}^{z} \leftrightarrow(-1)^{j} 2 \hat{S}_{j-1, j}^{z}, \hat{\sigma}_{j}^{x} \leftrightarrow\left(\hat{\Phi}_{j-1}^{\dagger} \hat{S}_{j-1, j}^{+} \hat{\Phi}_{j}+\right.$ H.c. $)$, $\hat{\sigma}_{j}^{y} \leftrightarrow-i(-1)^{j}\left(\hat{\Phi}_{j-1}^{\dagger} \hat{S}_{j-1, j}^{+} \hat{\Phi}_{j}-\right.$ H.c. $)$ and the parameters $\Omega=-w, \delta=-m$. This mapping can be applied both for open and periodic boundary conditions and it overcomes the most challenging task in quantum simulating gauge theories, by restricting the dynamics directly within the gauge-invariant Hilbert space. 
Compared to the opposite strategy of integrating out the gauge fields, our procedure based on integrating out matter degrees of freedom has major experimental implications. With the first approach, one would obtain linearly raising potentials which do not appear easily in the synthetic quantum systems, and lead to very large energy scales (of the order of the system size). Since the overall timescale of most experiments is limited by noise, having couplings with relative ratios of order $L$ is a severe limitation for analog experiments and partially affects also digital efforts. With our approach, the only states that would violate Gauss law are nearest-neighbor occupied sites which are strongly suppressed by the Rydberg blockade. Additional terms in the Hamiltonian, such as next-nearest neighbor interactions of Rydberg excitations, are mapped to gauge-invariant terms (e.g., next-nearest neighbor interactions between electric fields). From a theoretical viewpoint, the line of thought of our scheme is similar to the one used in hybrid Monte Carlo schemes, where one first integrates out the matter fields and then deals with a purely bosonic action.

Beyond providing a direct link between Gauss law and the Rydberg blockade mechanism, the most important feature of the mapping is that, differently from other remarkable relations between $\hat{H}_{\mathrm{FSS}}$ and lattice models with gauge symmetries [40,41], it provides an immediate connection between Rydberg experiments and particle physics phenomena, as we describe below.

\section{REAL-TIME DYNAMICS OF LATTICE GAUGE THEORIES IN RYDBERG ATOM EXPERIMENTS}

\section{A. Gauge-theory interpretation of slow dynamics}

The exact description of Rydberg-blockaded chains in terms of a U(1) LGT allows us to shed a new light on the slow dynamics reported in Ref. [14], by interpreting them in terms of well-studied phenomena in high-energy physics, related to the production of particle-antiparticle pairs after a quench akin to the Schwinger mechanism.

In the experiment, the system was initialized in a chargedensity wave state [CDW1 in Fig. 1(c)], and subsequently, the Hamiltonian was quenched, inducing slowly decaying oscillations between CDW1 and CDW2. As shown in Fig. 1(c), CDW1 and CDW2 are mapped onto the two states of the $S=1 / 2$ QLM with uniform electric field $\hat{S}_{j, j+1}^{z}= \pm 1 / 2$. The experimental results in Ref. [14] may thus be interpreted as the evolution starting from one of the two degenerate bare particle vacua $\left|0_{ \pm}\right\rangle$(i.e., the vacua in the absence of quantum fluctuations, $w=0$ ) of the gauge theory. In Fig. 1(d) and in the first column of Fig. 2, we illustrate these dynamics as it would be observed in the excitation density $\left\langle n_{j}\right\rangle$ along the Rydberg atom quantum simulators ("Rydberg") and compare it with that of the electric field $\left\langle E_{j, j+1}\right\rangle$ within its gauge-theory description ("Quantum link model") in Fig. 1(e) and in the second (a)

(b)

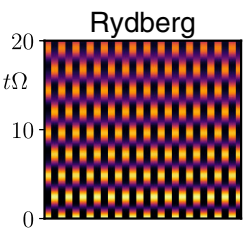

Quantum link

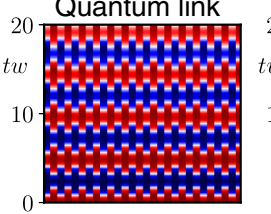

Schwinger

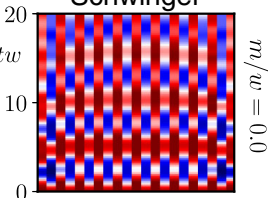

$t \Omega$
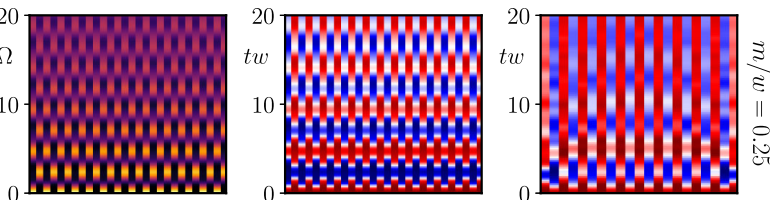

(c) 2

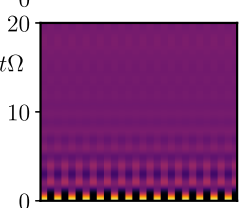

(d)
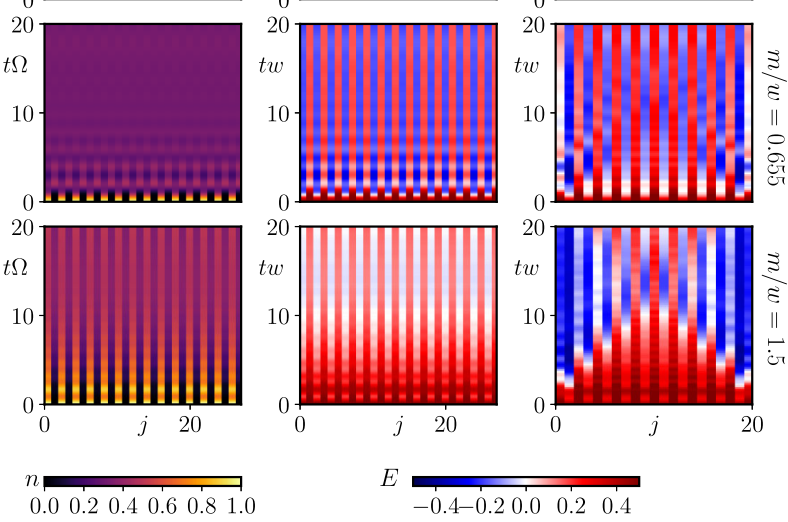

FIG. 2. Slow dynamics in Rydberg atoms, the U(1) quantum link model (QLM), and the lattice Schwinger model. Coherent quantum evolution of the local Rydberg excitation density profile (first column) $n_{j}(t)=\left\langle\hat{n}_{j}(t)\right\rangle$ in the FSS model [see Eq. (2)], starting from a charge-density wave, of the local electric field profile (second column) $E_{j, j+1}(t)=\left\langle\hat{S}_{j, j+1}^{z}(t)\right\rangle$ in the QLM, and (third column) $\left\langle\hat{L}_{j, j+1}(t)-\theta /(2 \pi)\right\rangle$ (see main text) in the lattice Schwinger model [see Eq. (3)] with $J / w=1.5$ and $\theta=\pi$. The four rows correspond to increasing values of the detuning $\delta$ (Rydberg) or, equivalently, of the particles mass $m=-\delta$ (QLM and Schwinger model). Figures 1(d) and 1(e) correspond to the first two plots in (a) here. Data in the first and second columns are connected by a unitary transformation, while a remarkable similarity is manifest between the second and third column despite the larger Hilbert space of the gauge degrees of freedom in the Schwinger model. The persistent string inversions observed within the symmetric phase with $m<m_{c}=0.655|w|$ in rows (a) and (b) are suppressed as the quantum critical point is approached. The dynamics in the third column feature edge effects due to the imposed open boundary conditions.

column of Fig. 2, respectively, utilizing exact diagonalization [42].

The qualitative features of this evolution are strongly affected by quantum fluctuations, whose impact is quantified by the ratio between the coupling constant $w$ and the particles mass $m$. For small values of $m / w$ [Figs. 2(a) and 2 (b)], production of particle-antiparticle pairs occurs at a finite rate. We remark that this effect is reminiscent of the Schwinger mechanism [6], which, however, concerns pair creation from the true (and not the bare) vacuum. These particles get accelerated by the electric field and progressively screen it, until coherent pair annihilation takes place 
and eventually brings the system to a state with opposite electric flux. This process, referred to as string inversion, occurs several times in a coherent fashion; similarly to what is observed in string-breaking scenarios (e.g., in other LGTs [26,43]), this causes a dramatic slowdown of thermalization and of quantum information propagation. As a further evidence, we compute both the total electric flux and the vacuum persistence amplitude (or Loschmidt echo), defined as $G_{+}(t)=\left|\left\langle 0_{+}\left|e^{-i \hat{H} t}\right| 0_{+}\right\rangle\right|^{2}$, whose large value $\simeq 1$ was already noted in Ref. [44]. The anomalous long-lived oscillations of these quantities experimentally detected with Rydberg atom arrays in Ref. [14] show a clear analogy with several previous numerical studies of the realtime dynamics of higher-spin QLMs [26] as well as of the Schwinger model $[24,25,45]$ and Higgs theories [21]. In addition, as noted in Ref. [38], the dynamics discussed here describes the coherent oscillations of the parity-symmetric order parameter (in our case, $\left\langle\hat{E}_{j, j+1}\right\rangle$ ) as a function of time, reminiscent of the decay of a chiral condensate in QCD [27]. We thus provide here a bridge among all these observations.

However, if fermionic particles are sufficiently heavy, with $m / w$ exceeding a critical threshold, pair production is a virtual process and string inversion cannot be triggered, as shown in Figs. 2(c) and 2(d). We find that this behavior is related to the quantum phase transition occurring in the FSS model at $\delta_{c}=-0.655|\Omega|$ [36]. This transition corresponds to the spontaneous breaking of the chiral symmetry in the LGT Eq. (4) at $m_{c}=0.655|w|$ [46]. Figure 2 shows the temporal evolution of the same initial uniform flux configuration [CDW or "string" in Fig. 1(c)] upon increasing values of the mass $m / w=0,0.25,0.655,1.5$ corresponding to the dynamics at $m<m_{c}$ [Figs. 2(a) and 2(b)], at the quantum critical point $m=m_{c}$ [Fig. 2(c)], and at $m>m_{c}$ [Fig. 2(d)].

Figure 3 further illustrates the appearance of string inversions for $m<m_{c}$ and the corresponding slow dynamics. Figure 3(a) shows the long-lived revivals of the manybody wave function in terms of the evolution of the probability $G_{ \pm}(t)$ of finding the system at time $t$ in the initial bare vacuum state $\left|0_{+}\right\rangle$or in the opposite one $\left|0_{-}\right\rangle$, corresponding to $G_{+}$or $G_{-}$, respectively, as well as in terms of the time-dependent density $\rho$ of particle-antiparticle pairs. The entanglement entropy of half system also displays an oscillatory behavior (see Appendix A). Figure 3(b) shows the scaling of the collective oscillations of the electric field with respect to the system size $L$, as well as their persistence with a small but nonvanishing fermion mass $m<m_{c}$.

\section{B. Slow dynamics in the Schwinger model}

The above phenomenology is not restricted to QLMs, but is expected to be a generic feature of LGTs including dynamical matter. We show this in the context of a Wilsonian LGT, i.e., the lattice version of the Schwinger model in Eq. (3). As discussed below, the model dynamics
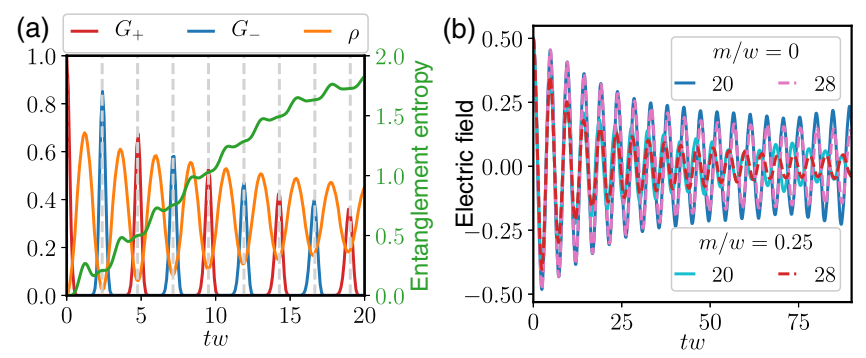

FIG. 3. Characterization of slow dynamics in the FSS model. (a) Hilbert space characterization of the persistent string inversions ( $m=0, L=28)$ : alternating strong revivals of the overlaps $G_{ \pm}(t)=\left|\left\langle 0_{ \pm}\left|e^{-i \hat{H} t}\right| 0_{+}\right\rangle\right|^{2}$ with the two bare vacuum states $\left|0_{ \pm}\right\rangle$, corresponding to the two charge-density wave configurations of Rydberg atom arrays. Both the total density $\rho=\left\langle\hat{\rho}_{j}\right\rangle$ of particleantiparticle pairs, with $\hat{\rho}_{j}=(-1)^{j} \hat{\Phi}_{j}^{\dagger} \hat{\Phi}_{j}+\left[1-(-1)^{j}\right] / 2$, and the half-chain entanglement entropy (see the Appendix A) have regularly spaced maxima between the peaks. (b) Persistent oscillations of electric field for two values of the mass and of the system size.

is, at the lattice level, remarkably different from the FSS model (no constraints when written in spin language, different Hilbert space scaling, different interactions, etc.). The key aspect is, instead, the common field-theoretical framework.

In this case, $\hat{U}_{j, j+1}=e^{i \hat{\vartheta}_{j, j+1}}$ are $\mathrm{U}(1)$ parallel transporters with vector potential $\hat{\vartheta}_{j, j+1}$, and the corresponding electric field operator is $\hat{E}_{j, j+1}=\hat{L}_{j, j+1}-\theta /(2 \pi)$, where $\hat{L}_{j, j+1}$ have integer spectrum and $\theta /(2 \pi)$ represents a uniform classical background field parametrized by the $\theta$ angle. Canonical commutation relations for the gauge degrees of freedom read $\left[\hat{\vartheta}_{j, j+1}, \hat{L}_{p, p+1}\right]=i \delta_{j p}$. In our numerical simulations, we utilize the spin formulation of the model obtained upon integration of the gauge fields under open boundary conditions $[47,48]$.

We consider the case of a $\theta$ angle with $\theta=\pi$, such that the electric field $\hat{E}_{j, j+1}$ has half-integer spectrum. Then, in the limit $J / w \rightarrow \infty$, the term $J \hat{E}_{j, j+1}^{2}$ in the Hamiltonian suppresses all the values of the electric field that are different from $\pm 1 / 2$. This implies that the electric field can be represented by a spin- $1 / 2 \hat{S}^{z}$ operator and that the lattice Schwinger model is equivalent to the spin-1/2 QLM discussed above. We find evidence that the corresponding behavior persists qualitatively down to $J \simeq w$, when the electrostatic energy competes with the matter-field interaction, as shown in the third column of Fig. 2. Despite the strong quantum fluctuations allowed in principle by the exploration of a locally infinite-dimensional Hilbert space, a qualitative similarity with the case of the locally finitedimensional Hilbert space of the QLM is manifest in the second column of Fig. 2, related to the observed dynamics in Ref. [14]. At a more quantitative level, we see that the periods of the oscillations in the lattice Schwinger model 

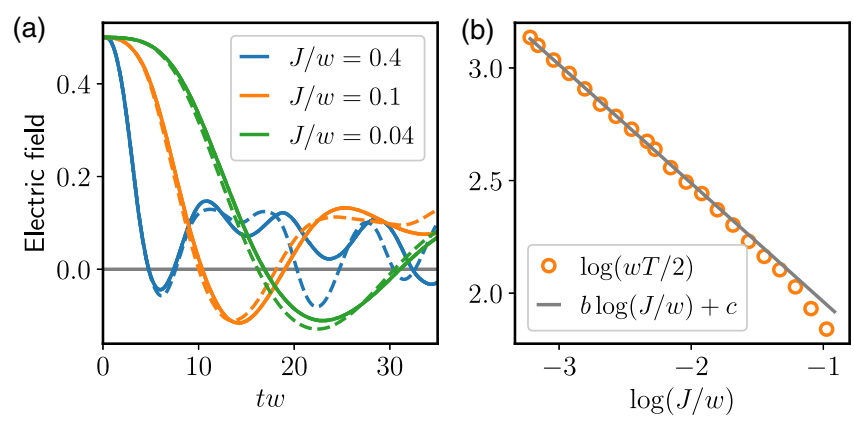

FIG. 4. Oscillation of the electric field in the Schwinger model with $\theta=\pi$. (a) Time evolution of the average electric field. The initial state is the bare vacuum with $E_{j, j+1}=1 / 2$ and the chain has periodic boundary conditions. The solid and dashed lines correspond to $L=14$ and $L=12$, respectively. Exact simulations are performed via truncation of the local Hilbert space to dimension 16, i.e., $\left|E_{j, j+1}\right|<15 / 2$, and the constrained Hamiltonian for the electric field is obtained by eliminating matter degrees of freedom. (b) Period $T$ of the oscillations as a function of $J$ and $w$. Data points correspond to values of the logarithm of the half period $T$ obtained for $L=14$. The solid line is the function $b \log (J / w)+c$, where $b=-0.526$ and $c=4.2$ are obtained through a fit in the region $J / w<0.1$.

and in the QLM (for the same couplings) are in good agreement.

Even more drastically, we observe persistent oscillations also down to $J \ll w$ (see Fig. 4), a regime in which the period becomes longer upon decreasing $J$. As we discuss below, the reasons why such oscillations persist for values of the parameters at the opposite regime with respect to the constrained one is related to the fieldtheoretical origin of such behavior, which can be even captured at a quantitative level via simple analytical approximations.

We remark that the lattice Schwinger model with unbounded levels of the gauge fields is substantially different from the QLM: not only the Hilbert space is much larger, but also the effective spin-1/2 model describing it (see Appendix C) features long-range Coulomb interactions. Therefore, the generality of the occurrence of oscillations which do not decay on timescales immediately related to the microscopic couplings points to a rather robust underlying mechanism. In fact, we suggest here that this behavior may arise from a universal field-theoretical description of the nonequilibrium dynamics of states possessing a well-defined continuum limit.

Concerning the U(1) LGTs discussed in this work, the reference continuum field-theory description is provided by the Schwinger model, representing quantum electrodynamics in one spatial dimension [16]. In the massless limit $m=0$, this model can be exactly mapped by bosonization to a free scalar bosonic field theory [6]. For a nonzero mass, the model is described in terms of the canonically conjugate fields $\hat{\Pi}$ and $\hat{\phi}$ by the Hamiltonian

$$
\begin{aligned}
\hat{H}_{B}= & \int d x\left[\frac{1}{2} \hat{\Pi}^{2}+\frac{1}{2}\left(\partial_{x} \hat{\phi}\right)^{2}+\frac{1}{2} \frac{e^{2}}{\pi} \hat{\phi}^{2}\right. \\
& \left.-c m \omega_{0} \cos (2 \sqrt{\pi} \hat{\phi}-\theta)\right] .
\end{aligned}
$$

Within this bosonized description, the field $\hat{\phi}(x, t)$ represents the electric field, and for $m=0$ all its Fourier modes $\tilde{\phi}(k)$ correspond to decoupled harmonic oscillators. In this case, the evolution starting from a false vacuum with a uniform string of nonvanishing electric field $\langle\hat{\phi}(x, t=0)\rangle=$ const $\neq 0$ represents an excitation of the single uniform mode with $k=0$, and hence the electric field will show uniform periodic string inversions around zero, with a frequency $\omega_{0}=e / \sqrt{\pi}$, where $e$ is the charge of the fermion. A nonvanishing value of $m$ leads to the additional anharmonic term in Eq. (5). The resulting total potential shows a transition from a shape with a single minimum for $m<m_{c}$ to two symmetric minima for $m>m_{c}$, analogous to the spontaneous breaking of chiral symmetry on the lattice (see the Appendix C for details). The weak local nonlinearity introduced by a small $m$ couples the various Fourier modes and hence induces a weak integrability breaking. In this case, the uniform string inversions of the electric field evolving from a false vacuum configuration with $\langle\tilde{\phi}(k=0)\rangle \neq 0$ are expected to be superseded by slow thermalization processes at long times (see, e.g., Ref. [49]). In the context of cold gases, a reminiscent slow relaxation has been observed in interfering bosonic Luttinger liquids, whose Hamiltonian dynamics has some similarities to the one discussed here [50].

We suggest that a remnant of this slow dynamics induced by the underlying integrable field theory may persist in lattice versions of this gauge theory as long as initial states with a well-defined continuum limit are considered. With the latter, we mean states whose field configuration is smooth at the level of the lattice spacing: for our case here, the two Néel states represent the smoother ones, as they correspond to the bare vacuum of the fermionic fields, and no electric field excitations. At a qualitative level, the effect of integrability breaking induced by lattice effects is expected to be much weaker in the small Hilbert space sector involving uniform excitations with $k=0$ only, where the long-lived string inversion dynamics take place. The number of states in this sector grows linearly with the lattice size $L$, and their energy spans an extensive range of the spectrum, in agreement with the characteristics of "many-body quantum scars"; see Ref. [28] and Sec. IV D.

At a quantitative level, we test our prediction on the lattice Schwinger model with $\theta=\pi$ and $m=0$, whose continuum limit is obtained by scaling the parameters with the lattice spacing $a$ in such a way that $J=e^{2} a / 2$, $w=1 /(2 a)$, and $a \rightarrow 0$ [51]. In order to address this regime, we perform a scaling analysis as a function of $J / w$. According to the field theory, in this limit the period $T$ of 

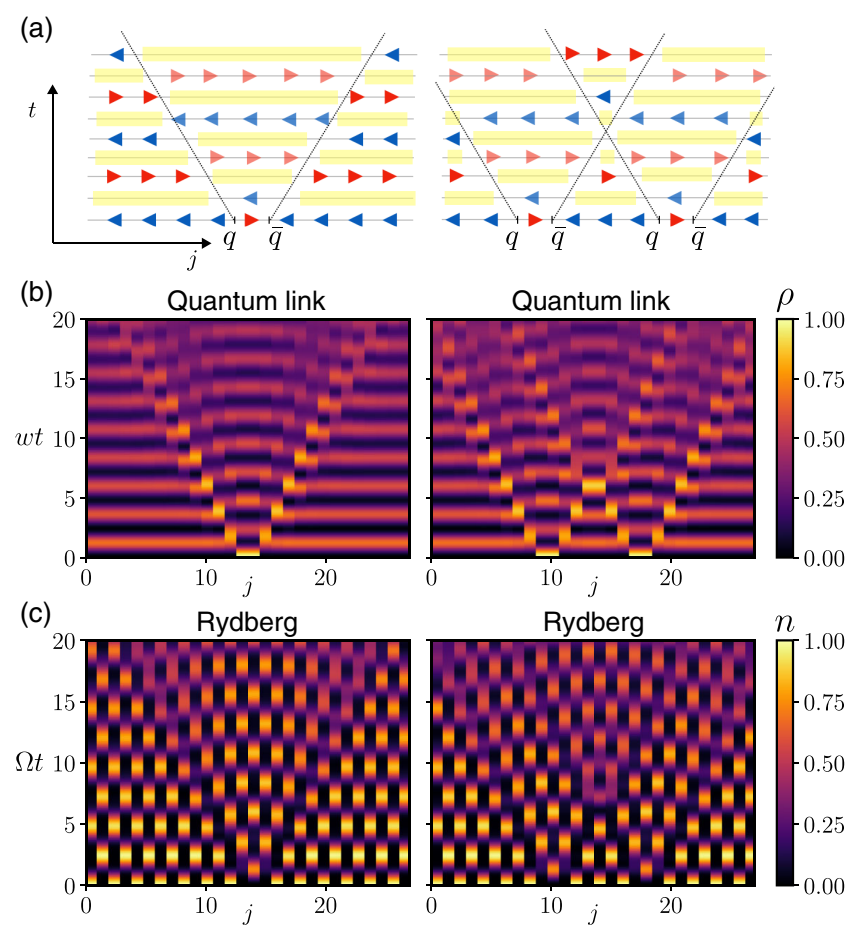

FIG. 5. Slow dynamics of particle-antiparticle pairs. (a) Illustration of the states involved in the propagation of particleantiparticle pairs $q-\bar{q}$. The notation is the same as in Fig. 1(c), while the yellow stripes denote regions of space with largest particle density and therefore $\left\langle\hat{E}_{j, j+1}\right\rangle \simeq 0$. (b): Evolution of the particle density in the QLM starting from a bare vacuum or "string" state, see Fig. 1(c), with initial particle-antiparticle pairs. (c) Same as in (b), but in the Rydberg excitation density representation. Left-hand column: The oscillations observed in the light-cone-shaped region originating from the particles is observed to be out of phase with respect to those of the bare vacuum. Right-hand column: In the presence of two $q-\bar{q}$ pairs, an additional change of periodicity is expected in correspondence of elastic scattering. In these simulations, $m=\delta=0$.

the oscillations scales as $T \propto 1 / \sqrt{J w}$ : as shown in Fig. 4, this scaling is indeed satisfied for $J \ll w$, where we obtain a fitting dependence of $T \propto(J w)^{-0.526}$ within a few percent form the expected exponent.

\section{Propagation of particle-antiparticle pairs}

States of the QLM corresponding to particle-antiparticle pairs in the bare vacuum can be constructed in Rydberg atom quantum simulators by preparing two or more defects in a charge-density wave configuration, each corresponding to pairs of adjacent nonexcited Rydberg atoms.

As an illustration, we discuss how the time evolution of one or two particle-antiparticle pairs for $m<m_{c}$ features the emergence of slow dynamics. In Fig. 5, we show the time evolution of both the particle density in the QLM and the corresponding density of excitations in the Rydberg chain, fixing for simplicity $m=0$. The pairs in the initial
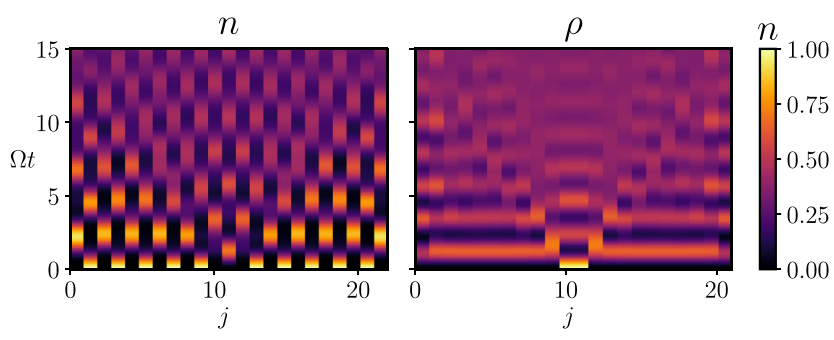

FIG. 6. Propagation of a particle-antiparticle pair $q-\bar{q}$ with realistic Rydberg interactions. Left-hand panel: Density of Rydberg excitations. Right-hand panel: Density of particles or antiparticles ( $\rho$ in the QLM language). Results are obtained for a chain of $L=23$ sites governed by the realistic Hamiltonian Eq. (1) with $V_{i j}=V_{1}|i-j|^{-6}$ and no constraints in the Hilbert space. Parameters are $\delta=0, V_{1} / \Omega=25.6$. We checked explicitly that the violation of Rydberg blockade is always small, with $\left\langle n_{j} n_{j+1}\right\rangle<10^{-2}$.

state break and ballistic spreading of quark and antiquark takes place. The string inversion dynamics induced by this propagation shows coherent interference patterns with longlived oscillations. Because of retardation effects induced by the constrained dynamics, these oscillations are shifted by half a period with respect to the vacuum oscillation, as captured by second-order perturbation theory.

These unusual dynamics turn out to be robust under experimentally realistic conditions: In Fig. 6, we consider the evolution of a particle-antiparticle pair, the simulated dynamics of which is not constrained to the subspace satisfying $\hat{n}_{j} \hat{n}_{j+1}=0$ but includes the effect of the longrange Rydberg interactions between atoms. The evolution is performed via Krylov subspace techniques in the unconstrained Hilbert space with the Hamiltonian in Eq. (1), with $\delta=0$ and $V_{j, k}=V_{1}|j-k|^{-6}$. The value of $V_{1} / \Omega=25.6$ is the same as considered in Ref. [14]. The dynamics displayed in Fig. 6 is similar to the constrained one in Figs. 5(b) and 5(c) at short times, after which the effects of having realistic interactions gradually kick in.

\section{Spectral properties and bands of non-thermal states}

We characterize the anomalous ballistic spreading of particle-antiparticle pairs discussed in the previous section in terms of the emergence of corresponding anomalous spectral properties of the FSS model, which generalize those recently observed [28] in the special case $m=0$, involving families of special energy eigenstates referred to as "many-body quantum scars." The latter are constituted by towers of regularly spaced states in the many-body spectrum with alternating pseudomomentum $k=0$ and $k=\pi$, characterized by nonthermal expectation values of local observables as well as by anomalously large overlaps with the charge-density wave initial states. The long-lived coherent oscillating behavior has been attributed in Ref. [28] to the existence of these "scarred" eigenstates. 


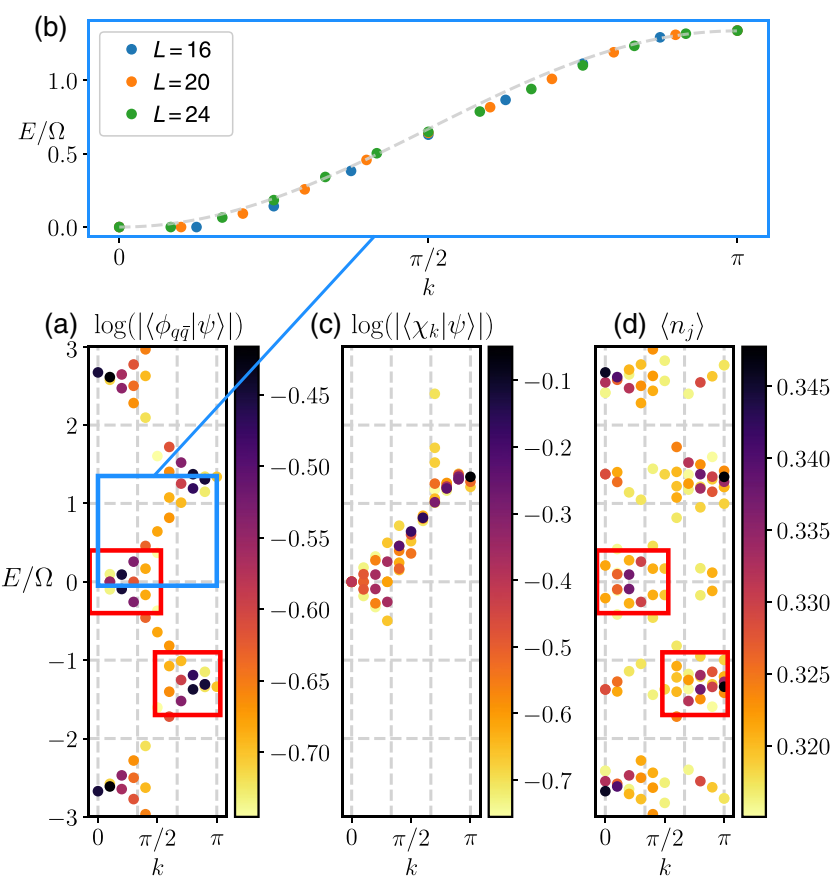

FIG. 7. Emergent quasiparticle description of highly excited states. (a) Largest overlaps of the initial state $\left|\phi_{q \bar{q}}\right\rangle$ with a localized defect in a charge-density wave configuration of the Rydberg atom chain with the energy eigenstates $|\psi\rangle$ of the FSS Hamiltonian $(\delta=0, L=20)$ in Eq. (2), as a function of their corresponding momentum and energy. Within the gauge-theory description, the initial state corresponds to having a localized particle-antiparticle pair $q-\bar{q}$. (b) The eigenstates with the largest overlaps display a regular functional dependence of energy on momentum that is remarkably close to a simple cosine band. (c) The largest overlaps of the optimal matrix-product state quasiparticle ansatz $\left|\chi_{k}\right\rangle$ built on an exact eigenstate with zero energy (see the main text) accurately reproduce the corresponding emergent quasiparticle band of (a). (d) Anomalous (nonthermal) expectation values of a local observable in energy eigenstates. The red boxes highlight the correspondence between the most relevant eigenstates building up $\left|\phi_{q \bar{q}}\right\rangle$ (a) and the most nonthermal eigenstates (d). The emergent spectral structure illustrated here underlies the clean ballistic spreading of particleantiparticle pairs displayed in Fig. 5.

Figure 7(a) shows that the modulus of the overlap between the energy eigenstate $|\psi\rangle$ with energy $E$ and the inhomogeneous states $\left|\phi_{q \bar{q}}\right\rangle$ indicating a particleantiparticle pair with momentum $k$ clearly identifies a number of special bands of highly excited energy eigenstates characterized each by an emerging functional relationship $E(k)$. As shown in Fig. 7(d), the occupation $\left\langle n_{j}\right\rangle$ of some of the states in these bands strongly deviate from the thermal value $\left\langle n_{j}\right\rangle_{\text {th }} \simeq 0.276$. This fact has already been observed in the previously studied quantum-scarred eigenstates, which coincide with the extremal points of these bands at momenta $k=0$ and $k=\pi$. A closer inspection of these energy-momentum relations, presented in Fig. 7(b), shows that they are close to cosine-shaped bands, suggesting the emergence of single-particle excitations in the middle of the manybody energy spectrum.

We further characterize this spectral structure by constructing a quasiparticle variational ansatz $\left|\chi_{k}\right\rangle$ on top of the exact matrix-product-state zero-energy eigenstate of the Hamiltonian (2) with $\delta=0$, recently put forward in Ref. [30] (see the Appendix B). As shown in Fig. 7(c), the optimal quasiparticle ansatz has the largest overlap with the states on the energy-momentum bands of special eigenstates closest to zero energy, thus reinforcing the above emergent quasiparticle picture.

\section{E. Tuning the topological $\theta$ angle in Rydberg experiments}

So far, our discussion has focused exclusively on the relation between Rydberg experiments and the Schwinger model with topological angle $\theta=\pi$. A natural question to ask is whether, within the present setting, it is possible to realize genuinely confining theories, i.e., generic values of the topological angle $\theta \neq \pi$.

This is possible within the strong coupling limit upon introducing a linear term in the electric field. With reference to the lattice Schwinger model introduced in Sec. IV B and notations therein, we see that the two lowest degenerate energy states of the local electric field for $\theta=\pi$ (i.e., $\left.L_{j, j+1}=0,+1\right)$ are split when $\theta$ deviates from $\pi$, with an energy gap $\Delta=J|\theta / \pi-1|$. In order to keep the structure of the Hilbert space compatible with the FSS model, one requires this $\Delta$ to be much smaller than the gaps with the other states, which are of the order of $J$. This implies that, within the QLM formulation, we can only access very small deviations from $\theta=\pi$ : this is not a limiting factor, and we will show how this simple setting already allows us to witness the effects of confinement in the dynamics. The confining nature of the potential can be intuitively understood as follows: starting from the bare vacuum (the "string" state in Fig. 1), creating and separating a particleantiparticle pair at a distance $\ell$ entails the creation, between the two, of a string of length $\ell$ with opposite electric field. The corresponding energy cost is proportional to $\ell \Delta$, signaling the confining nature of the potential. Accordingly, the lattice Schwinger model with strong $J \gg \Omega, m, \Delta$ and with a topological angle $\theta=$ $\pi(1 \pm \Delta / J)$ is efficiently approximated by the QLM with an additional term linear in the electric field and proportional to $\Delta$.

In turn, within the exact mapping outlined in Sec. III and illustrated in Fig. 1, this $\theta$-angle term corresponds to an additional staggered field in the FSS model, leading to the Hamiltonian:

$$
\hat{H}_{\mathrm{Ryd}}=\sum_{j=1}^{L}\left(\Omega \hat{\sigma}_{j}^{x}+\delta \hat{\sigma}_{j}^{z}\right)+\sum_{j=1}^{L}(-1)^{j} \frac{\Delta}{2} \sigma_{j}^{z} .
$$




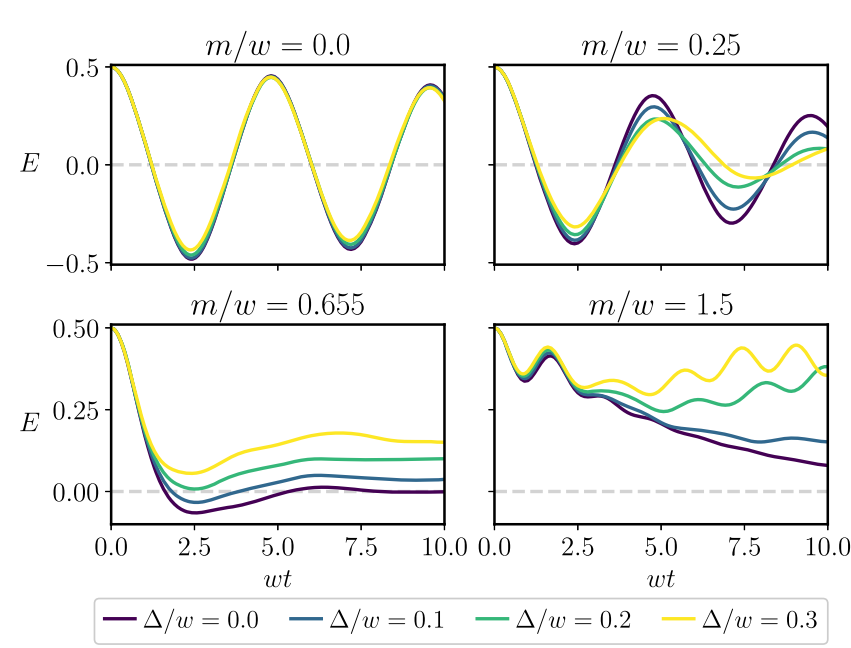

FIG. 8. Effect of the $\theta$ angle on the dynamics of the electric field from uniform string states of the QLM. Data are shown for a chain of $L=28$ sites, for increasing values of the particle mass $m / w$ and of the parameter $\Delta$, quantifying the deviation of the $\theta$ angle from $\pi$ (see the main text). Dynamics for $\Delta=0$ correspond to the second column of Fig. 2.

The new term can be experimentally realized, e.g., by utilizing a position-dependent ac Stark shift or, alternatively, a space-dependent detuning on the transition between ground and Rydberg states (it was realized, for example, in a recent work reported in Ref. [52]).

In Fig. 8 , we show the effect of the $\theta$ angle on the evolution of the total electric field in the QLM starting from a uniform string state. Also in this case, the dynamics observed can be understood using the bosonized field theory in Eq. (5). As explained in Sec. IV B, the integrability-breaking term which appears for $m>0$ has the effect of damping the oscillations. Moreover, from the same equation we can predict that the impact of a variation of the $\theta$ term on the dynamics is enhanced when we increase the mass, as data clearly show. This enhancement of the $\theta$ dependence becomes more evident when we cross the transition point: while in the symmetric phase with $m<m_{c}$, the explicit symmetry breaking caused by the electric field energy imbalance leads to damping of the string inversions, in the broken-symmetry (chiral) phase with $m>m_{c}$, the effect of confinement is dramatic, causing the persistence of the initial electric string, with small long-lived oscillations. Focusing on the latter phase, in Fig. 9 we show the dynamical evolution of a finite electric string generated by a particle-antiparticle pair (lefthand panels), at the deconfined point $\theta=\pi$ (top) and in the confined phase with $\theta \neq \pi$ (bottom). The right-hand panels show the same evolution as it would appear in terms of measurements of Rydberg atom excitations. While for $\Delta=0$ nothing prevents the initially localized bare particles to propagate along the chain (top panels), the presence of a linear confining potential proportional to $\Delta$ between them stabilizes the electric string, leading to effective Bloch
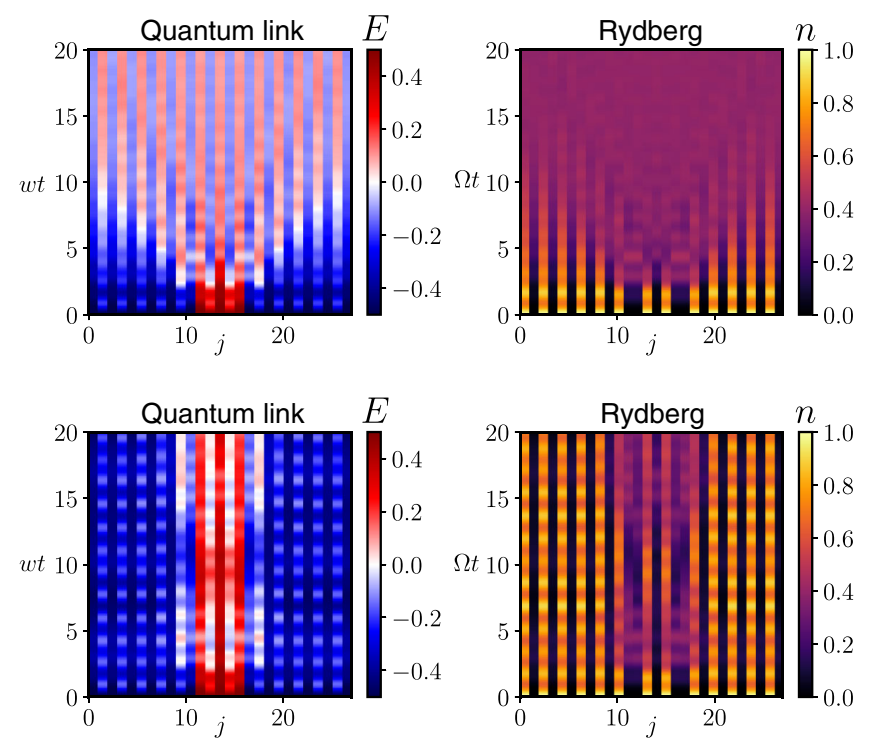

FIG. 9. $\quad \theta$ angle and string-breaking dynamics. The evolution of a bare particle-antiparticle pair state is displayed in terms of space- and time-dependent electric field in the QLM (left-hand panels) and of the density of excited atoms in the Rydberg array (right-hand panels), with $m=-\delta=1.5 \Omega$ and $L=28$. Simulations in the top row have $\Delta=0$, corresponding to the deconfined field theory with $\theta=\pi$. Effects of confinement emerge in the second row, where a nonvanishing $\Delta=0.3 \Omega$ stabilizes the electric string.

oscillations of the edges and to a surprisingly long lifetime [53] (bottom panels). This effect signals that confinement can dramatically affect the nonequilibrium dynamics, potentially slowing it down as observed in both gauge theories [54] and statistical mechanics models [53,55,56]. In this regime, the model shows the same qualitative signatures of confinement as the quantum Ising chain in transverse and longitudinal field: the long-lived coherent oscillations, the suppression of the light-cone spreading [55], and the presence of anomalous eigenstates [56].

\section{CONCLUSIONS}

We proved that the large-scale quantum simulation of lattice gauge theories has already been achieved in state-ofthe-art experiments with Rydberg atoms, as it can be realized by establishing a mapping between a U(1) gauge theory and Rydberg atom arrays. At the theoretical level, we showed that this novel interpretation provides additional insight into the exotic dynamics observed in experiments, linking it to archetypal phenomena in particle physics. Our field-theoretic description immediately implies the generality and applicability to a wide variety of model Hamiltonians within experimental reach, and among them we extensively discuss the example of the lattice Schwinger model in the Wilson formulation. We expect that future studies can further deepen the connection between the statistical mechanics description of such behavior and its 
gauge-theoretic interpretation, for instance, elucidating the effects of nonthermal states [28-31] and emergent integrability [32,33], and the role of confinement in slowing down the dynamics [53-57]. At the experimental level, our findings immediately motivate further experiments along this direction that can probe different aspects of gauge theories, such as the decay of unstable particle-antiparticle states after a quench, and might be combined with other quantum information protocols [58]. We show how by tuning the $\theta$ angle - a possibility that is already available with current technologies - the different dynamical regimes expected from the field theory can be accessed. A particularly interesting perspective in this direction is the possibility of dynamically probing confinement via quantum quenches starting from a string embedded in the (bare) vacuum, a prototypical gedanken experiment in particle physics [24].

The quantum simulation strategy we propose is based on the elimination of the matter degrees of freedom by exploiting Gauss law: This method does not rely on the specific formulation of the model and is in principle applicable to other lattice gauge theories (for a recent work along these lines see, e.g., Ref. [59]). An intriguing future extension is represented by theories with non-Abelian gauge symmetries, an example of which can be found in Ref. [60], where links with finite-dimensional Hilbert spaces are utilized. The integration of matter degrees of freedom is equally well suited for higher dimensions, and Rydberg atoms are a promising platform for pursuing this direction [61,62], with the additional advantage that the major complication in realizing non-Abelian theories (i.e., engineering complicated and fine-tuned Gauss laws) is replaced by considerably simpler dynamical constraints. After the present analysis, the experiments performed in Ref. [14] represent a stepping stone toward the ambitious realization of non-Abelian gauge theories in three spatial dimension, which remains an outstanding quest $[7,10]$.

\section{ACKNOWLEDGMENTS}

We thank M. Aidelsburger, J. Berges, P. Calabrese, M. Collura, A. Dabholkar, P. Hauke, R. Konik, Z. Papic, A. Rudra, and A. Scardicchio for fruitful discussions. M. D. thanks D. Banerjee, S. Montangero, E. Rico, U.-J. Wiese, and P. Zoller for collaboration on related works, and M. Lukin and H. Pichler for insightful discussions and correspondence. This work is partially supported by the ERC under Grant No. 758329 (AGEnTh), by the QUANTERA project QTFLAG, and has received funding from the European Union's Horizon 2020 research and innovation programme under Grant Agreement No. 817482.

\section{APPENDIX A: ENTANGLEMENT EVOLUTION IN THE FSS MODEL}

We consider the FSS model defined in Eq. (2) and we investigate the time evolution of the bipartite entanglement
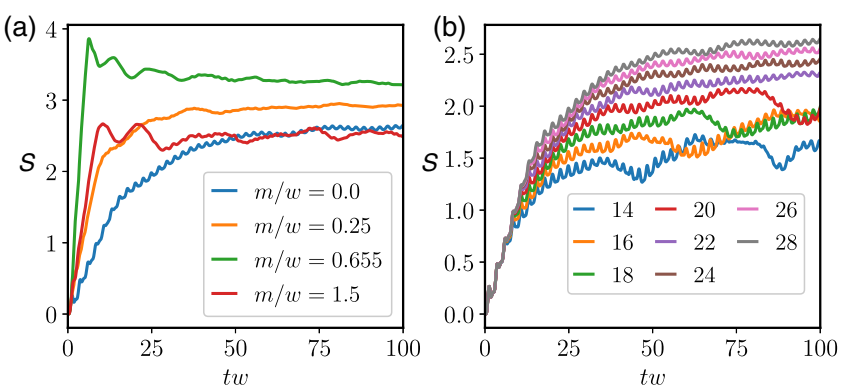

FIG. 10. Evolution of entanglement entropy in the FSS model. (a) Growth of the half-chain entanglement entropy for various values of the particle mass $m$. Initial state is CDW or, equivalently, a string, and $L=28$. (b) Growth of entanglement entropy for different sizes $L$. Initial state is $\mathrm{CDW}$ or, equivalently, a string, and $m=0$.

entropy $S(t)$ of the chain. We consider as initial state the $\mathrm{CDW}$, which is equivalent to considering the QLM evolving from one of the two uniform string configurations; see Fig. 1. In order to determine $S$, we compute the timedependent reduced density matrix $\hat{\rho}_{\mathrm{R}}(t)$ of a subsystem consisting of $L / 2$ consecutive sites of the chain, by tracing out the degrees of freedom of the remaining complementary $L / 2$ sites. In these terms, the von Neumann entanglement entropy is defined by $S(t)=-\operatorname{Tr}\left[\hat{\rho}_{\mathrm{R}}(t) \ln \hat{\rho}_{\mathrm{R}}(t)\right]$.

Figures 10(a) and 10(b) show the evolution of $S$ for various values of the mass $m$ and of the chain length $L$, respectively. Information spreading is directly tied to particle production: it is fast at the critical point $m=m_{c}$ [green curve in Fig. 10(a)] with $m_{c} / w=0.655$, or above it $m>m_{c}$ (red curve), where particles are not confined. For $m<m_{c}$ (yellow and blue curves), instead, it slows down considerably, as was already observed in the spin-1 QLM [26]. For $m / w=0$, the change in the original slope of the curve which occurs around $t w \simeq 12$ is due to a finite-volume effect, as demonstrated in Fig. 10(b), where such a change progressively disappears upon increasing $L$. In all cases, the fast oscillations correspond to different stages of pair production.

\section{APPENDIX B: SPECTRAL PROPERTIES OF THE FSS MODEL}

\section{Robustness of the spectral structure}

As shown in the main text, the FSS model for $m=0$ features the emergence of regular structures in the middle of the spectrum in terms of energy-momentum bands. We here show that these structures are generically present for sufficiently small values of $|m / w|$. Figure 11 shows the energy-momentum relation of the eigenstates which have the largest overlaps with the inhomogeneous state $\left|\phi_{q \bar{q}}\right\rangle$ defined in Sec. IV D. For $m / w=0.1$ and $m / w=-0.2$, similar dispersion relations to the case $m / w=0$ are observed, the main difference being an overall energy shift. 

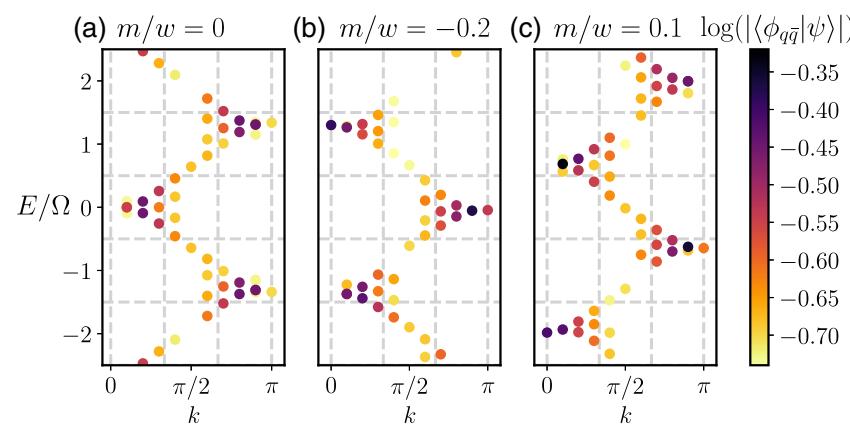

FIG. 11. Robustness of the spectral structure. Energy-momentum relation of eigenstates around $E=0$ for $L=20$. For each eigenstate $|\psi\rangle$, the color indicates the value of $\log _{10}\left(\left|\left\langle\psi \mid \phi_{q \bar{q}}\right\rangle\right|\right)$ (eigenstates with smallest overlaps are not plotted). The dispersion observed for $m / w=0$ in (a) is shifted but persists when we introduce a nonzero mass as in (b) and (c).

\section{Quasiparticle ansatz for emergent excitations}

In order to obtain physical intuition on the emergence of regular energy-momentum bands in highly excited states which govern the nonequilibrium evolution of localized defects, we propose the following wave function,

$$
\left|\chi_{k}\right\rangle=\sum_{j=1}^{L} e^{-i k j} \hat{O}_{j-1, j, j+1}\left|\Phi_{k=0}\right\rangle,
$$

where $\left|\Phi_{k=0}\right\rangle$ is the exact eigenstate found in Ref. [30] with momentum $k=0$ and energy 0 , and $\hat{O}_{j-1, j, j+1}$ is a threesite operator depending on a number of variational parameters. Because of the constraints, the space where this operator acts is reduced from dimension $2^{3}$ to 5 . The inversion symmetry with respect to site $j$ reduces the number or free variational parameters in $\hat{O}_{j-1, j, j+1}$ to 11 . We choose a basis of operators $\left\{\hat{M}_{j-1, j, j+1}^{\alpha}\right\}_{\alpha=1}^{11}$ for parametrizing $\hat{O}_{j-1, j, j+1}$ and define

$$
\left|\phi_{k}^{\alpha}\right\rangle=\sum_{j=1}^{L} e^{-i k j} \hat{M}_{j-1, j, j+1}^{\alpha}\left|\Phi_{k=0}\right\rangle .
$$

For each $k$, we minimize the energy variance in the space spanned by the states $\left|\phi_{k}^{\alpha}\right\rangle$. To this aim, we compute the three matrices $N_{\alpha \beta}^{k}=\left\langle\phi_{k}^{\alpha} \mid \phi_{k}^{\beta}\right\rangle, P_{\alpha \beta}^{k}=\left\langle\phi_{k}^{\alpha}|\hat{H}| \phi_{k}^{\beta}\right\rangle, Q_{\alpha \beta}^{k}=$ $\left\langle\phi_{k}^{\alpha}\left|\hat{H}^{2}\right| \phi_{k}^{\beta}\right\rangle$. In order to prevent numerical issues in the minimization, we diagonalize the matrix of the norms $N_{k}$ and we compute the (rectangular) matrix $U^{k}$ whose columns are the eigenvectors of $N^{k}$ having nonzero eigenvalues. We then find the vector $\boldsymbol{c}_{\boldsymbol{k}}=\left(c_{k}^{1}, \ldots, c_{k}^{m}\right)$ that minimizes

$$
\sigma_{\hat{H}}^{2}=\frac{\boldsymbol{c}_{\boldsymbol{k}}^{\dagger} U^{k \dagger} Q^{k} U^{k} \boldsymbol{c}_{\boldsymbol{k}}}{\boldsymbol{c}_{\boldsymbol{k}}^{\dagger} U^{k \dagger} N^{k} U^{k} \boldsymbol{c}_{\boldsymbol{k}}}-\left(\frac{\boldsymbol{c}_{\boldsymbol{k}}^{\dagger} U^{k \dagger} P^{k} U^{k} \boldsymbol{c}_{\boldsymbol{k}}}{\boldsymbol{c}_{\boldsymbol{k}}^{\dagger} U^{k \dagger} N^{k} U^{k} \boldsymbol{c}_{\boldsymbol{k}}}\right)^{2} .
$$

Note that by introducing the matrix $U^{k}$ we restrict the minimization to states with nonzero norms, thus further reducing the number of variational parameters to $m(k) \leq 11$. The optimal wave function is then obtained as

$$
\left|\chi_{k}\right\rangle=\sum_{\alpha=1}^{11} \sum_{\beta=1}^{m} U_{\alpha \beta}^{k} c_{k}^{\beta}\left|\phi_{k}^{\beta}\right\rangle .
$$

\section{APPENDIX C: DYNAMICS OF THE SCHWINGER MODEL}

\section{Mapping onto a long-range interacting spin chain}

The lattice Schwinger model in Eq. (3) in the gaugeinvariant subspace spanned by wave functions $|\psi\rangle$ which satisfy the Gauss laws $\hat{G}_{j}|\psi\rangle=0$ can be conveniently simulated by exactly mapping it onto an unconstrained chain of spin- $1 / 2$ degrees of freedom in the case of open boundary conditions [51]. These spins are obtained from the fermionic operators via a combination of a Jordan-Wigner transformation and a gauge transformation, expressed as

$$
\hat{\Phi}_{j}=\prod_{l=1}^{j-1}\left(\hat{\sigma}_{l}^{z} \hat{U}_{l, l+1}^{\dagger}\right) \hat{\sigma}_{j}^{-} .
$$

This transformation decouples spins and gauge degrees of freedom, and thus the Hamiltonian Eq. (3) takes the form

$$
\hat{H}=-w \sum_{j=1}^{L-1}\left(\hat{\sigma}_{j}^{+} \hat{\sigma}_{j+1}^{-}+\text {H.c. }\right)+\frac{m}{2} \sum_{j=1}^{L}(-1)^{j} \hat{\sigma}_{j}^{z}+J \sum_{j=1}^{L-1} \hat{E}_{j, j+1}^{2} .
$$

The electric field can be rewritten in terms of the spin operators and of the background field $\alpha$ by means of the Gauss law:

$$
\hat{E}_{j, j+1}=\frac{1}{2} \sum_{l=1}^{j}\left[\hat{\sigma}_{l}^{z}+(-1)^{l}\right]-\alpha .
$$

Inserting Eq. (C3) into Eq. (C2), we obtain three additional terms: a long-range spin-spin interaction corresponding to a Coulomb interaction, a local energy offset that modifies the effective mass of the fermions, and a linear potential given by the constant background field. The electric field part of the Hamiltonian can be cast in the form:

$$
\begin{aligned}
\hat{H}_{\mathrm{lat}}^{E}= & \frac{J}{2} \sum_{n=1}^{L-2} \sum_{l=n+1}^{L-1}(L-l) \hat{\sigma}_{n}^{z} \hat{\sigma}_{l}^{z}-\frac{J}{4} \sum_{n=1}^{L-1}\left[1-(-1)^{n}\right] \sum_{l=1}^{n} \hat{\sigma}_{l}^{z} \\
& -J \alpha \sum_{j=1}^{L-1}(L-j) \hat{\sigma}_{j}^{z} .
\end{aligned}
$$


In this form, the nonequilibrium dynamics of the lattice Schwinger model can be efficiently simulated with standard algorithms of quantum many-body physics.

The origin of long-range spin-spin interactions as a consequence of the linear confining Coulomb potential in one spatial dimension is made more evident when Eq. (C4) is formulated in terms of the charges $\hat{Q}_{j}=$ $\left[\hat{\sigma}_{j}^{z}+(-1)^{j}\right] / 2[63]$. In the neutral charge sector where $\sum_{j=1}^{L} \hat{Q}_{j}=0$, we have

$$
\begin{aligned}
\hat{H}_{\text {lat }}^{E}= & -J \sum_{j=1}^{L-1} \sum_{k=j+1}^{L}(k-j) \hat{Q}_{j} \hat{Q}_{k}-J \sum_{j=1}^{L}(L+1-j) \alpha \hat{Q}_{j} \\
& +J \sum_{j=1}^{L} j \alpha \hat{Q}_{j} .
\end{aligned}
$$

The first term describes the Coulomb interaction between charges, while the remaining two terms can be interpreted as interactions with two static charges $-\alpha$ and $\alpha$, placed at the boundaries of the chain (sites 0 and $L+1$, respectively) and effectively producing the constant background field.

\section{Continuum limit of the massive Schwinger model}

The massive Schwinger model briefly introduced in the main text describes the quantum electrodynamics of fermions of mass $m$ and charge $e$ in $1+1$ dimensions. Its Lagrangian density is

$$
\mathcal{L}=-\frac{1}{4} F_{\mu \nu} F^{\mu \nu}+\bar{\psi}(i \not \supset-e \not A-m) \psi
$$

where $F_{\mu \nu}=\partial_{\mu} A_{\nu}-\partial_{\mu} A_{\nu}$ and $A_{\mu}$ is the vector potential. The indices $\mu, \nu=0,1$ indicate, respectively, the time and space directions, and the slash notation indicates contraction with the Dirac matrices $\gamma_{\mu}$. This model can be formulated in terms of a bosonic field $\phi$ [47]. We briefly recall here the main points of the derivation of the bosonic Hamiltonian obtained in Ref. [16].

In the Coulomb gauge $\left(A_{1}=0\right)$, the Euler-Lagrange equation for $A_{0}$ yields

$$
\partial_{1}^{2} A_{0}=-e j_{0},
$$

where $j_{0}=\psi^{\dagger} \psi$ is the charge density. Integrating Eq. (C7), we obtain the continuum version of Eq. (C3),

$$
F_{01}=-\partial_{1} A_{0}=e \partial_{1}^{-1} j_{0}+F
$$

where $F$ is a number, representing a classical background field. The Hamiltonian density obtained from the Lagrangian Eq. (C6) has the form

$$
\mathcal{H}=\bar{\psi}\left(i \gamma_{1} \partial_{1}+m\right) \psi+\frac{1}{2} F_{01}^{2}
$$

The interacting Hamiltonian for the fermions can be formulated using Eq. (C8) to integrate out the gauge fields. Integrating by parts in the zero charge sector, i.e., $\int d x j_{0}(x)=0$, we obtain

$$
\begin{aligned}
H= & \int d x \bar{\psi}\left(i \gamma_{1} \partial_{1}+m\right) \psi-\frac{e^{2}}{4} \int d x d y j_{0}(x) j_{0}(y)|x-y| \\
& -e F \int d x x j_{0}(x) .
\end{aligned}
$$

Similarly to the lattice version of this model [cf. Eqs. (C2) and (C5)], the resulting Hamiltonian contains the energy of massive free fermions, the Coulomb interaction between charges (which increases linearly in one spatial dimension), and the interactions between the charges and the background field.

The method of bosonization can be applied, by noting that in $1+1$ dimensions the conserved vector field $j^{\mu}=$ $\bar{\psi} \gamma^{\mu} \psi$ can be written as the curl of a scalar field $\phi$ :

$$
j_{\mu}=\pi^{-1 / 2} \epsilon_{\mu \nu} \partial^{\nu} \phi
$$

By substituting in Eq. (C8), we get

$$
F_{01}=e \pi^{-1 / 2} \phi+F,
$$

and, from the results obtained for a free massive Dirac field [64], we know

$$
\begin{aligned}
\bar{\psi}\left(i \gamma_{1} \partial_{1}+m\right) \psi \rightarrow & N_{m}\left[\frac{1}{2} \Pi^{2}+\frac{1}{2}\left(\partial_{1} \phi\right)^{2}\right. \\
& \left.-c m^{2} \cos \left(2 \pi^{1 / 2} \phi\right)\right]
\end{aligned}
$$

where $c=e^{\gamma} /(2 \pi), \quad \gamma \simeq 0.577$ is the Euler constant, and $N_{m}$ indicates normal ordering with respect to the mass $m$. Inserting Eqs. (C12) and (C13) in Eq. (C9), the Hamiltonian density reads

$$
\begin{aligned}
\mathcal{H}= & N_{m}\left[\frac{1}{2} \Pi^{2}+\frac{1}{2}\left(\partial_{1} \phi\right)^{2}-c m^{2} \cos \left(2 \pi^{1 / 2} \phi\right)\right. \\
& \left.+\frac{e^{2}}{2 \pi}\left(\phi+\frac{\pi^{1 / 2} F}{e}\right)^{2}\right] .
\end{aligned}
$$

By shifting the field $\phi \rightarrow \phi-\pi^{1 / 2} F / e$ and defining a new normal ordering with respect to the mass $\mu=\pi^{-1 / 2} e$, we finally obtain 
$\mathcal{H}=N_{\mu}\left[\frac{1}{2} \Pi^{2}+\frac{1}{2}\left(\partial_{1} \phi\right)^{2}-c m \mu \cos \left(2 \pi^{1 / 2} \phi-\theta\right)+\frac{\mu^{2}}{2} \phi^{2}\right]$,

(C15)

where $\theta=2 \pi F / e$. The latter form connects with the discussion in the main text—cf. Eq. (5) therein.

[1] K. G. Wilson, Confinement of Quarks, Phys. Rev. D 10, 2445 (1974).

[2] I. Montvay and G. Muenster, Quantum Fields on a Lattice (Cambridge University Press, Cambridge, England, 1994).

[3] Z. Fodor and C. Hoelbling, Light Hadron Masses from Lattice QCD, Rev. Mod. Phys. 84, 449 (2012).

[4] K. Fukushima and T. Hatsuda, The Phase Diagram of Dense QCD, Rep. Prog. Phys. 74, 014001 (2011).

[5] R. A. Soltz, C. DeTar, F. Karsch, S. Mukherjee, and P. Vranas, Lattice QCD Thermodynamics with Physical Quark Masses, Annu. Rev. Nucl. Part. Sci. 65, 379 (2015).

[6] E. A. Calzetta and B. L. Hu, Nonequilibrium Quantum Field Theory (Cambridge University Press, Cambridge, England, 2008).

[7] U. J. Wiese, Ultracold Quantum Gases and Lattice Systems: Quantum Simulation of Lattice Gauge Theories, Ann. Phys. (Amsterdam) 525, 777 (2013).

[8] E. Zohar, I. I. Cirac, and B. Reznik, Quantum Simulations of Lattice Gauge Theories Using Ultracold Atoms in Optical Lattices, Rep. Prog. Phys. 79, 014401 (2016).

[9] M. Dalmonte and S. Montangero, Lattice Gauge Theories Simulations in the Quantum Information Era, Contemp. Phys. 57, 388 (2016).

[10] J. Preskill, Simulating Quantum Field Theory with a Quantum Computer, arXiv:1811.10085.

[11] E. A. Martinez, C. A. Muschik, P. Schindler, D. Nigg, A. Erhard, M. Heyl, P. Hauke, M. Dalmonte, T. Monz, P. Zoller et al., Real-Time Dynamics of Lattice Gauge Theories with a Few-Qubit Quantum Computer, Nature (London) 534, 516 (2016).

[12] S. Trotzky, Y-A. Chen, A. Flesch, I. P. McCulloch, U. Schollwöck, J. Eisert, and I. Bloch, Probing the Relaxation towards Equilibrium in an Isolated Strongly Correlated One-Dimensional Bose Gas, Nat. Phys. 8, 325 (2012).

[13] I. Bloch, J. Dalibard, and S. Nascimbène, Quantum Simulations with Ultracold Quantum Gases, Nat. Phys. 8, 267 (2012).

[14] H. Bernien, S. Schwartz, A. Keesling, H. Levine, A. Omran, H. Pichler, S. Choi, A. S. Zibrov, M. Endres, M. Greiner et al., Probing Many-Body Dynamics on a 51-Atom Quantum Simulator, Nature (London) 551, 579 (2017).

[15] D. Barredo, V. Lienhard, S. de Léséleuc, T. Lahaye, and A. Browaeys, Synthetic Three-Dimensional Atomic Structures Assembled Atom by Atom, Nature (London) 561, 79 (2018).

[16] S. Coleman, More about the Massive Schwinger Model, Ann. Phys. (N.Y.) 101, 239 (1976).

[17] G. S. Bali, H. Neff, T. Düssel, T. Lippert, and K. Schilling (SESAM Collaboration), Observation of String Breaking in QCD, Phys. Rev. D 71, 114513 (2005).
[18] F. Hebenstreit, J. Berges, and D. Gelfand, Real-Time Dynamics of String Breaking, Phys. Rev. Lett. 111, 201601 (2013).

[19] S. Chandrasekharan and U. J. Wiese, Quantum Link Models: A Discrete Approach to Gauge Theories, Nucl. Phys. B492, 455 (1997).

[20] Y. Kuno, K. Kasamatsu, Y. Takahashi, I. Ichinose, and T. Matsui, Real-Time Dynamics and Proposal for Feasible Experiments of Lattice Gauge-Higgs Model Simulated by Cold Atoms, New J. Phys. 17, 063005 (2015).

[21] Y. Kuno, S. Sakane, K. Kasamatsu, I. Ichinose, and T. Matsui, Quantum Simulation of $(1+1)$-Dimensional U(1) Gauge-Higgs Model on a Lattice by Cold Bose Gases, Phys. Rev. D 95, 094507 (2017).

[22] J. Schwinger, Gauge Invariance, and Mass. II, Phys. Rev. 128, 2425 (1962).

[23] J. Kogut and L. Susskind, Hamiltonian Formulation of Wilson's Lattice Gauge Theories, Phys. Rev. D 11, 395 (1975).

[24] F. Hebenstreit, J. Berges, and D. Gelfand, Simulating Fermion Production in $1+1$ Dimensional QED, Phys. Rev. D 87, 105006 (2013).

[25] B. Buyens, J. Haegeman, H. Verschelde, F. Verstraete, and K. Van Acoleyen, Confinement and String Breaking for $\mathrm{QED}_{2}$ in the Hamiltonian Picture, Phys. Rev. X 6, 041040 (2016).

[26] T. Pichler, M. Dalmonte, E. Rico, P. Zoller, and S. Montangero, Real-Time Dynamics in U(1) Lattice Gauge Theories with Tensor Networks, Phys. Rev. X 6, 011023 (2016).

[27] K. Rajagopal and F. Wilczek, Emergence of Coherent Long Wavelength Oscillations after a Quench: Application to QCD, Nucl. Phys. B404, 577-589 (1993).

[28] C. J. Turner, A. A. Michailidis, D. A. Abanin, M. Serbyn, and Z. Papić, Weak Ergodicity Breaking from Quantum Many-Body Scars, Nat. Phys. 14, 745 (2018).

[29] C. J. Turner, A. A. Michailidis, D. A. Abanin, M. Serbyn, and Z. Papić, Quantum Scarred Eigenstates in a Rydberg Atom Chain: Entanglement, Breakdown of Thermalization, and Stability to Perturbations, Phys. Rev. B 98, 155134 (2018).

[30] C.-J. Lin and O. I. Motrunich, Exact Quantum Many-Body Scar States in the Rydberg-Blockaded Atom Chain, Phys. Rev. Lett. 122, 173401 (2019).

[31] W. W. Ho, S. Choi, H. Pichler, and M. D. Lukin, Periodic Orbits, Entanglement and Quantum Many-Body Scars in Constrained Models: Matrix Product State Approach, Phys. Rev. Lett. 122, 040603 (2019).

[32] S. Choi, C. J. Turner, H. Pichler, W. W. Ho, A. A. Michailidis, Z. Papić, M. Serbyn, M. D. Lukin, and D. A. Abanin, Emergent SU(2) Dynamics and Perfect Quantum Many-Body Scars, Phys. Rev. Lett. 122, 220603 (2019).

[33] V. Khemani, C. R. Laumann, and A. Chandran, Signatures of Integrability in the Dynamics of Rydberg-Blockaded Chains, Phys. Rev. B 99, 161101(R) (2019).

[34] I. Lesanovsky and H. Katsura, Interacting Fibonacci Anyons in a Rydberg Gas, Phys. Rev. A 86, 041601(R) (2012).

[35] J. Zeiher, J. y. Choi, A. Rubio-Abadal, T. Pohl, R. van Bijnen, I. Bloch, and C. Gross, Coherent Many-Body Spin 
Dynamics in a Long-Range Interacting Ising Chain, Phys. Rev. X 7, 041063 (2017).

[36] P. Fendley, K. Sengupta, and S. Sachdev, Competing Density-Wave Orders in a One-Dimensional Hard-Boson Model, Phys. Rev. B 69, 075106 (2004).

[37] D. Horn, Finite Matrix Models with Continuous Local Gauge Invariance, Phys. Lett. B 100, 149 (1981).

[38] D. Banerjee, M. Dalmonte, M. Müller, E. Rico, P. Stebler, U.-J. Wiese, and P. Zoller, Atomic Quantum Simulation of Dynamical Gauge Fields Coupled to Fermionic Matter: From String Breaking to Evolution after a Quench, Phys. Rev. Lett. 109, 175302 (2012).

[39] The similarity between the phenomenology of the two models was pointed out in Ref. [38]. Here, we are instead interested in establishing an exact relation.

[40] B. M. McCoy and M.-L. Yan, Gauge-Invariant Correlation Functions for the Ising-Gauge Ising-Higgs System in 2 Dimensions, Nucl. Phys. B215, 278 (1983).

[41] N. Chepiga and F. Mila, DMRG Investigation of Constrained Models: From Quantum Dimer and Quantum Loop Ladders to Hard-Boson and Fibonacci Anyon Chains, SciPost Phys. 6, 033 (2019).

[42] Exact diagonalization is performed on the gauge invariant subspace: for large $L$, its dimension grows as $\phi^{L}$, where $\phi$ is the golden ratio, thus allowing for large system sizes.

[43] T. Chanda, J. Zakrzewski, M. Lewenstein, and L. Tagliacozzo, Confinement and Lack of Thermalization after Quenches in the Bosonic Schwinger Model, arXiv:1909.12657 [Phys. Rev. Lett. (to be published)].

[44] Y.-P. Huang, D. Banerjee, and M. Heyl, Dynamical Quantum Phase Transitions in U(1) Quantum Link Models, Phys. Rev. Lett. 122, 250401 (2019).

[45] V. Kasper, F. Hebenstreit, M. Oberthaler, and J. Berges, Schwinger Pair Production with Ultracold Atoms, Phys. Lett. B 760, 742 (2016).

[46] E. Rico, T. Pichler, M. Dalmonte, P. Zoller, and S. Montangero, Tensor Networks for Lattice Gauge Theories and Atomic Quantum Simulation, Phys. Rev. Lett. 112, 201601 (2014).

[47] C. J. Hamer, J. Kogut, D. P. Crewther, and M. M. Mazzolini, The Massive Schwinger Model on a Lattice: Background Field, Chiral Symmetry and the String Tension, Nucl. Phys. B208, 413 (1982).

[48] M. C. Bañuls, K. Cichy, I. Cirac, and K. Jansen, The Mass Spectrum of the Schwinger Model with Matrix Product States, J. High Energy Phys. 11 (2013) 158.

[49] F. Fucito, F. Marchesoni, E. Marinari, G. Parisi, L. Peliti, S. Ruffo, and A. Vulpiani, Approach to Equilibrium in a Chain of Nonlinear Oscillators, J. Phys. (Paris) 43, 707 (1982).

[50] T. Langen, T. Gasenzer, and J. Schmiedmayer, Prethermalization and Universal Dynamics in Near-Integrable Quantum Systems, J. Stat. Mech. (2016) 064009.
[51] T. Banks, L. Susskind, and J. Kogut, Strong-Coupling Calculations of Lattice Gauge Theories: $(1+1)$-Dimensional Exercises, Phys. Rev. D 13, 1043 (1976).

[52] A. Omran, H. Levine, A. Keesling, G. Semeghini, T. T. Wang, S. Ebadi, H. Bernien, A. S. Zibrov, H. Pichler, S. Choi et al., Generation and Manipulation of Schrödinger Cat States in Rydberg Atom Arrays, Science 365, 570 (2019).

[53] P. P. Mazza, G. Perfetto, A. Lerose, M. Collura, and A. Gambassi, Suppression of Transport in Nondisordered Quantum Spin Chains due to Confined Excitations, Phys. Rev. B 99, 180302(R) (2019).

[54] M. Brenes, M. Dalmonte, M. Heyl, and A. Scardicchio, Many-Body Localization Dynamics from Gauge Invariance, Phys. Rev. Lett. 120, 030601 (2018).

[55] M. Kormos, M. Collura, G. Takács, and P. Calabrese, Real Time Confinement Following a Quantum Quench to a NonIntegrable Model, Nat. Phys. 13, 246 (2017).

[56] A. J. A. James, R. M. Konik, and N. J. Robinson, Nonthermal States Arising from Confinement in One and Two Dimensions, Phys. Rev. Lett. 122, 130603 (2019).

[57] J. Park, Y. Kuno, and I. Ichinose, Glassy Dynamics from Quark Confinement: Atomic Quantum Simulation of the Gauge-Higgs Model on a Lattice, Phys. Rev. A 100, 013629 (2019).

[58] C. Kokail, C. Maier, R. van Bijnen, T. Brydges, M. K. Joshi, P. Jurcevic, C. A. Muschik, P. Silvi, R. Blatt, C. F. Roos et al., Self-Verifying Variational Quantum Simulation of the Lattice Schwinger Model, Nature (London) 569, 355 (2019).

[59] S. Notarnicola, M. Collura, and S. Montangero, Real-TimeDynamics Quantum Simulation of $(1+1)$-Dimensional Lattice QED with Rydberg Atoms, Phys. Rev. Research 2, 013288 (2020).

[60] E. Zohar and J. I. Cirac, Removing Staggered Fermionic Matter in $U(n)$ and $S U(n)$ Lattice Gauge Theories, Phys. Rev. D 99, 114511 (2019).

[61] A. W. Glaetzle, M. Dalmonte, R. Nath, I. Rousochatzakis, R. Moessner, and P. Zoller, Quantum Spin Ice and Dimer Models with Rydberg Atoms, Phys. Rev. X 4, 041037 (2014).

[62] A. Celi, B. Vermersch, O. Viyuela, H. Pichler, M. D. Lukin, and P. Zoller, Emerging 2D Gauge Theories in Rydberg Configurable Arrays, arXiv:1907.03311.

[63] P. Sala, T. Shi, S. Kühn, M. C. Bañuls, E. Demler, and J. I. Cirac, Variational Study of $U(1)$ and $S U(2)$ Lattice Gauge Theories with Gaussian States in $1+1$ Dimensions, Phys. Rev. D 98, 034505 (2018).

[64] S. Coleman, Quantum Sine-Gordon Equation as the Massive Thirring Model, Phys. Rev. D 11, 2088 (1975). 